\title{
miR-2909-mediated regulation of KLF4: a novel molecular mechanism for differentiating between B-cell and T-cell pediatric acute lymphoblastic leukemias
}

Deepti Malik', Deepak Kaul ${ }^{1,3^{*}}$, Nalini Chauhan ${ }^{1}$ and Ram Kumar Marwaha ${ }^{2}$

\begin{abstract}
Background: microRNAs (miRNAs) play both oncogenic and oncostatic roles in leukemia. However, the molecular details underlying miRNA-mediated regulation of their target genes in pediatric B- and T-cell acute lymphoblastic leukemias (ALLs) remain unclear. The present study investigated the relationship between miR-2909 and Kruppel-like factor $4(K L F 4)$, and its functional relevance to cell cycle progression and immortalization in patients with pediatric ALL.

Methods: Elevated levels of miR-2909 targeted the tumor suppressor gene KLF4 in pediatric B-cell, but not pediatric T-cell ALL, as detected by pMIR-GFP reporter assay. Expression levels of genes including apoptosis-antagonizing transcription factor (AATF), MYC, B-cell lymphoma (BCL3), P2 ${ }^{C I P}, C C N D 1$ and SP1 in B- and T-cells from patients with pediatric ALL were compared with control levels using real-time quantitative reverse transcription polymerase chain reaction, western blotting, and reporter assays.

Results: We identified two novel mutations in KLF4 in pediatric T-ALL. A mutation in the $3^{\prime}$ untranslated region of the KLF4 gene resulted in loss of miR-2909-mediated regulation, while mutation in its first or third zinc-finger motif (Zf1/Zf3) rendered KLF4 transcriptionally inactive. This mutation was a frameshift mutation resulting in alteration of the Zf3 motif sequence in the mutant KLF4 protein in all pediatric T-ALL samples. Homology models, docking studies and promoter activity of its target gene $P 21^{C I P}$ confirmed the lack of function of the mutant KLF4 protein in pediatric T-ALL. Moreover, the inability of miR-2909 to regulate KLF4 and its downstream genes controlling cell cycle and apoptosis in T-cell but not in B-ALL was verified by antagomiR-2909 transfection. Comprehensive sequence analysis of KLF4 identified the predominance of isoform $1(\sim 55 \mathrm{kDa})$ in most patients with pediatric B-ALL, while those with pediatric T-ALL expressed isoform $2(\sim 51 \mathrm{kDa})$.
\end{abstract}

Conclusions: This study identified a novel miR-2909-KLF4 molecular axis able to differentiate between the pathogeneses of pediatric B- and T-cell ALLS, and which may represent a new diagnostic/prognostic marker.

Keywords: Acute lymphoblastic leukemia, miR-2909, Kruppel-like factor 4, Homology modeling, Cell cycle

\footnotetext{
* Correspondence: dkaul_24@hotmail.com

'Department of Experimental Medicine \& Biotechnology, Postgraduate Institute of Medical Education \& Research, Chandigarh, India

${ }^{3}$ Department of Experimental Medicine \& Biotechnology, Research Block B,

Postgraduate Institute of Medical Education \& Research, Chandigarh 160012,

India

Full list of author information is available at the end of the article
} 


\section{Background}

Acute lymphoblastic leukemia (ALL) is widely recognized as the most prevalent pediatric leukemia [1]; however, the genomic mechanisms responsible for the uncontrolled cell proliferation coupled with cell immortalization remain unknown [2]. In this context, the genes for apoptosisantagonizing transcription factor $(A A T F)$ and Kruppel like factor 4 (KLF4) have assumed importance. AATF provides a critical link between cell cycle progression, check-point control, and apoptosis [3], and also encodes the novel microRNA (miRNA) miR-2909, which regulates genes involved in inflammation, cell cycle, and immune response [4-6]. KLF4, a member of the SP1/KLF transcription factor family, is characterized by three highly conserved $\mathrm{C} 2 \mathrm{H} 2-$ type zinc-finger motifs at its carboxyl terminus, which are crucial for its interaction with its target DNA [7]. The KLF4 gene acts as both an oncogene and a tumor suppressor, depending on its genetic and cellular contexts [8]. The tumor-suppressive role of KLF4 and its involvement in regulating apoptosis, proliferation, and differentiation in Bcell malignancies suggest that KLF4 may play a critical role in leukemogenesis [9]. Furthermore, KLF4 mRNA has been shown to be targeted by miR-130a and 135b in M1 acute myeloid leukemic blasts, and silencing of KLF4 arrested the maturation of blood cells at an early progenitor stage [10].

The discovery of miRNAs has opened a new epigenomic dimension in terms of the understanding of oncogenesis in general and leukemogenesis in particular [11]. Alterations in miRNA expression patterns and their respective targets have been documented in various tumors [12] including different types of leukemias such as chronic lymphocytic leukemia [13], acute myeloid leukemia [14] and ALL [15], thus suggesting a possible correlation between miRNA expression status and the development of hematological malignancies.

The present study aimed to identify the expression status of AATF-encoded miR-2909 in B- and T-cells from patients with pediatric ALL and explore the possible relationship between miR-2909 and KLF4 in these cells. We also investigated the functional importance of this relationship in the regulation of genes involved in cell cycle progression (BCL3, CCND1, MYC) and apoptosis $(A A T F)$. To the best of our knowledge, the results of this study provide the first evidence for miR-2909-dependent regulatory pathway as the possible underlying mechanism responsible for the initiation of ALL in humans.

\section{Results}

\section{miR-2909 targets KLF4}

Quantitative real-time polymerase chain reaction (PCR) analysis of miR-2909 expression in 30 pediatric patients with B-ALL and 20 with T-ALL revealed significantly increased expression levels compared with the corresponding controls (Figure 1A). To investigate the molecular mechanism of miR-2909 function in ALL, we performed in silico screening of genes reported to play crucial roles in leukemogenesis for the presence of miR-2909 target site(s) using an RNA hybrid tool (http://bibiserv.techfak.unibielefeld.de/rnahybrid/) [16]. Among all the identified genes, we focused on KLF4 because it was reported to be significantly downregulated in ALL and functions as a tumor suppressor in B-cell hematological malignancies [9]. The 3' untranslated region (UTR) region of KLF4 harbored a target site for miR-2909 (Figure 1B). To validate this prediction experimentally, KLF4 expression was observed at both the mRNA and protein levels in patients with ALL. Increased miR-2909 expression was always accompanied by significant downregulation of KLF4 mRNA and protein in pediatric B-ALL compared with controls, indicating that miR-2909 may regulate the expression of KLF4 by targeting its 3'UTR (Figure $1 \mathrm{C}$ and D). In contrast, both mRNA and protein expression levels of $K L F 4$ were upregulated in T-ALL compared with controls (Figure $1 \mathrm{C}$ and $\mathrm{D}$ ), despite increased expression of miR2909 in these T-ALL lymphoblasts (Figure 1A), suggesting the possibility of a mutation in either the seed sequence or the 3'UTR region of KLF4 involving the miR-2909 binding site.

Melting-curve graphs for miR-2909 found no difference between T-ALL and controls, thus ruling out the presence of a mutation in the seed sequence of miR2909 (Figure 1E). However, the melting-curve graph corresponding to the KLF4 3'UTR did show a significant difference (Figure 1F). In order to clarify these changes, amplicons corresponding to this region were sequenced and revealed the presence of a genetic aberration in the miR-2909-binding site of KLF4 in T-ALL (Figure 2A). In contrast, no aberration was depicted in the miR2909-binding site within the 3'UTR of KLF4 in B-ALL (Figure 2B). To further confirm if miR-2909 targets KLF4 in pediatric B-ALL but not in pediatric T-ALL, we constructed reporter plasmids using the KLF4 3'UTR containing the miR-2909 target site from B-ALL (pGFPKLF4-3'UTR-B) and T-ALL (pGFP-KLF4-3'UTR-T) patients (Figure 2D). The 3'UTR regions of the KLF4 genes from both B-ALL and T-ALL were amplified and inserted into the cloning site of the vector downstream of the green fluorescent protein (GFP) reporter gene. These plasmids were then transfected into HEK 293 cells, which intrinsically overexpressed miR-2909 compared with HeLa, PC3 and HepG2 cells (Figure 2C). HEK 293 cells carrying pGFP-KLF4-3'UTR-B exhibited a $52.07 \%$ reduction in GFP expression, whereas cells expressing pGFP-KLF4-3'UTR-T showed no noticeable difference compared with cells transfected with pGFP plasmid without 3'UTR inserts (Figure 2E and F). These results clearly revealed the ability of miR-2909 to repress KLF4 expression in pediatric B-ALL, but not T-ALL. 


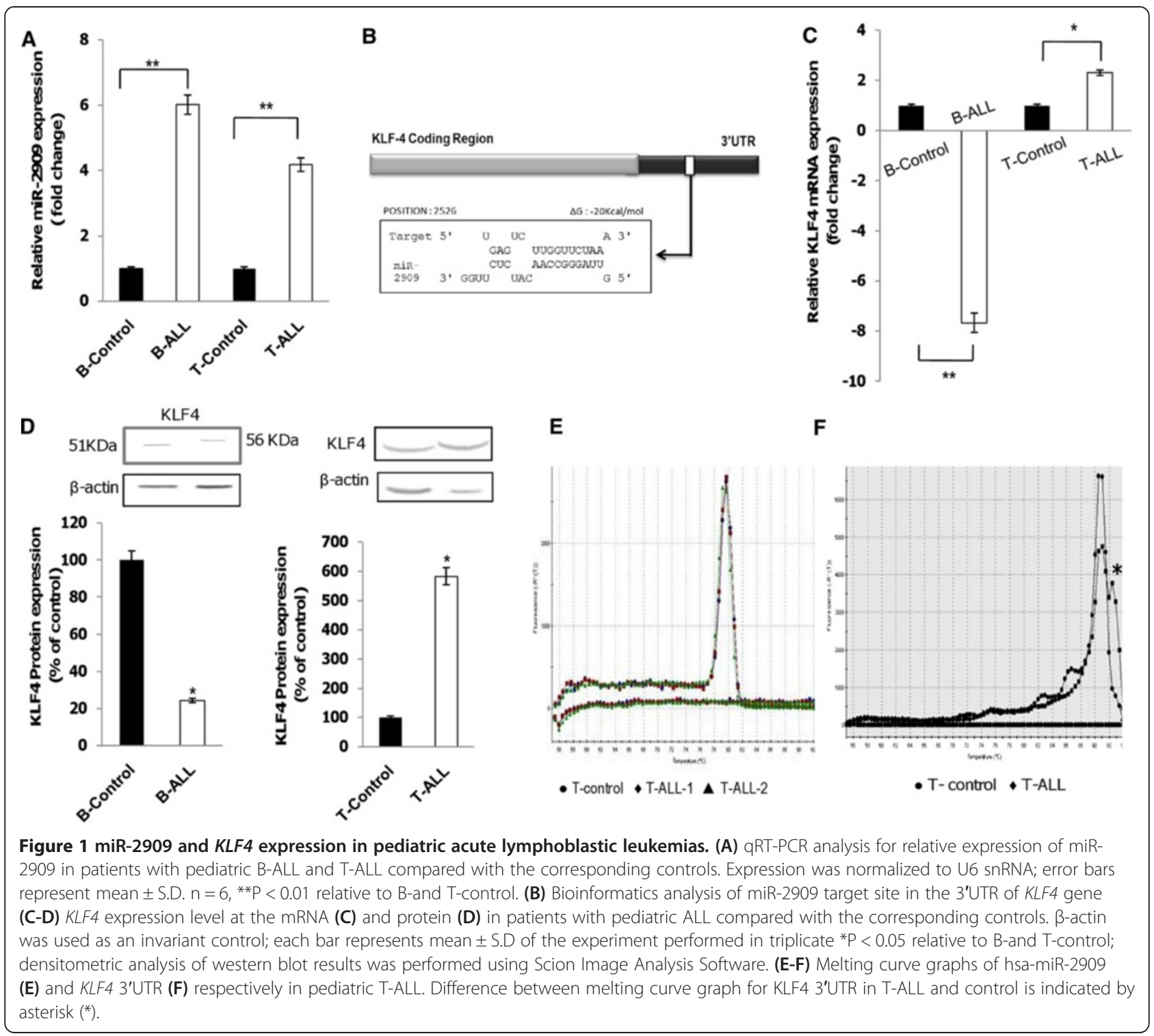

\section{Sequence analysis of KLF4}

UniProt gives three different isoforms of KLF4. Isoform 1 has the addition of 33 amino acids at location 367-400 while isoform 3 has a deletion of the first 50 amino acids (http://www.uniprot.org/uniprot/O43474) [17]. We aimed to determine if the isoforms of KLF4 differed between B-ALL and T-ALL. The amplicons corresponding to fulllength KLF4 were sequenced in 30 B-ALL and 20 T-ALL samples. Strikingly, 25 B-ALL samples showed the presence of isoform 1, which incorporated an intronic region between exons 3 and 4 in the coding sequence resulting in an increased molecular mass of approximately $55 \mathrm{kDa}$ (total length of 513 amino acids). The remaining five BALL and all $20 \mathrm{~T}$-ALL samples showed the presence of isoform 2, containing 479 amino acids with a molecular mass of $51 \mathrm{kDa}$ (Figure $3 \mathrm{~A}$ and $\mathrm{B}$ ).
We further explored if T-ALL patients expressed isoform 3 of $K L F-4$. Sequencing failed to identify any deletion of the first 50 amino acids in any of the T-ALL samples in the present study, confirming the absence of isoform 3 in these blast cells (Figure 3C). Interestingly, most T-ALL samples showed insertion/deletion of nucleotides in the region corresponding to the third zincfinger ( $\mathrm{Zf} 3$ ) motif of KLF4, while deletion of nucleotides in the first zinc-finger (Zf1) motif was identified in some T-ALL samples (Figure 3D, Additional file 1: Figure S1). These genetic aberrations changed the entire reading frame, altering the sequence of the KLF4 zinc-finger motif and potentially destroying its DNA-binding affinity (Additional file 1: Figure S1G). Modeling/docking studies were performed using mutant KLF4 lacking the Zf3 motif but with intact Zf1 and Zf2 motifs derived from 


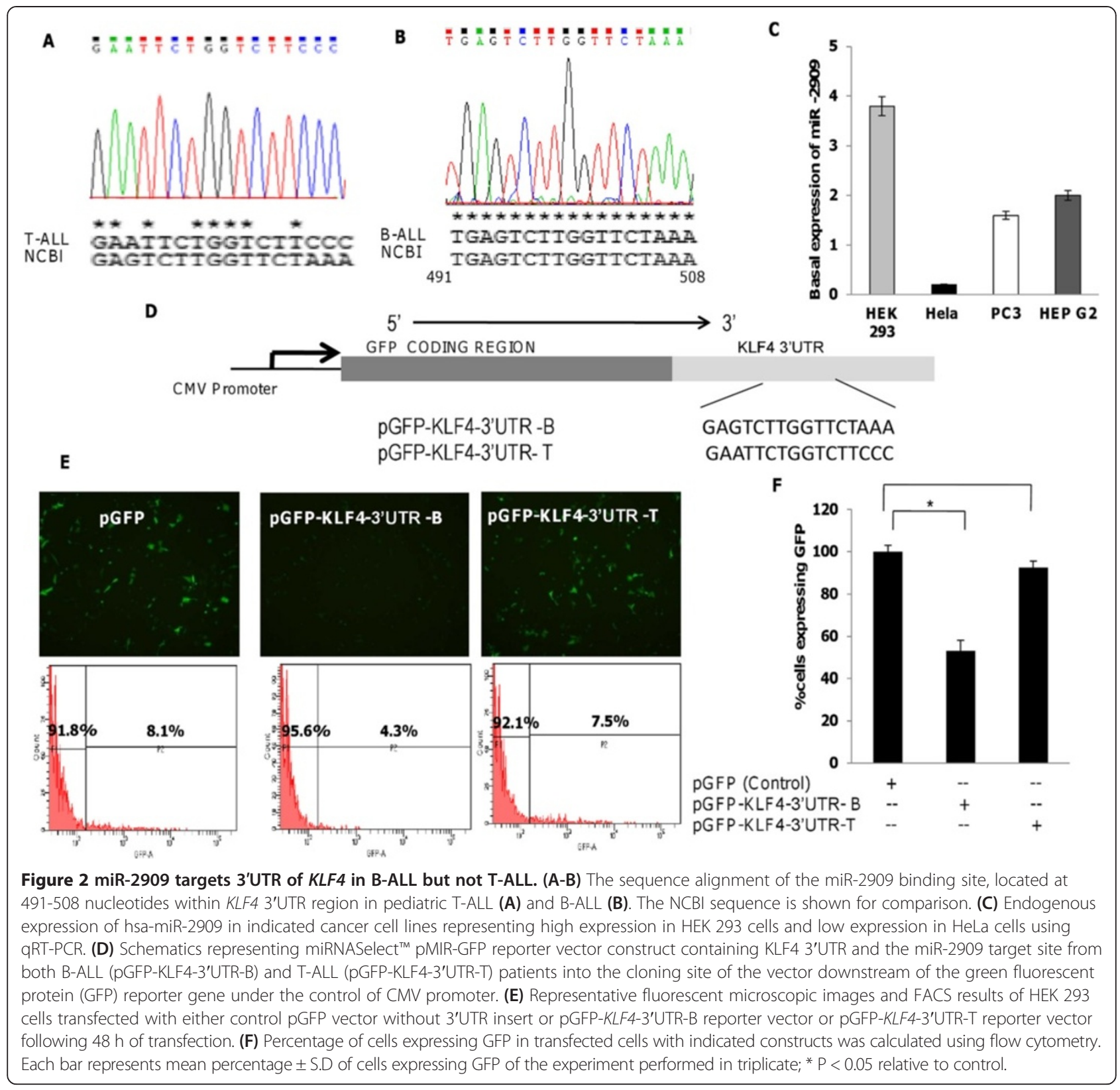

pediatric T-ALL samples (Figure 3E). In addition, we also sequenced the KLF4 coding region corresponding to the three zinc-finger motifs in B-ALL samples to detect the presence of any genetic aberration(s) in this region in pediatric B-ALL. Sequence analysis revealed no genetic aberrations in any of the three zinc-fingers regions of KLF4 in samples from pediatric patients with B-ALL, suggesting that the conformation of KLF4 was unaffected in these patients (Additional file 2: Figure S2).

Molecular modeling and docking studies of KLF4 protein Sequence alignment of wild-type KLF4 (derived from control T cells) with mutant KLF4 (derived from pediatric
T-ALL samples) revealed that the Zf3 motif was mutated (Figure 4A). Comparison of structural models of the third zinc-finger motif between wild-type and mutant KLF4 revealed the amino acid replacements $C 462 \mathrm{~V}, \mathrm{C} 465 \mathrm{M}$, and $\mathrm{H} 482 \mathrm{~F}$, which were involved in coordination with zinc (Figure 4B, C and D). The structure of wild-type KLF4 revealed the existence of a salt bridge between the guanidinium group of arginine residues (R449 and R471) and the surrounding anionic carboxylate $\left(\mathrm{RCOO}^{-}\right)$side chains of glutamic and aspartic acid (E446 and D473), respectively. The third glutamate residue (E446) formed a salt bridge with cationic ammonium $\left(\mathrm{RNH}_{3}^{+}\right)$of lysine (E446-L453). In contrast, in mutant KLF4, the guanidinium 


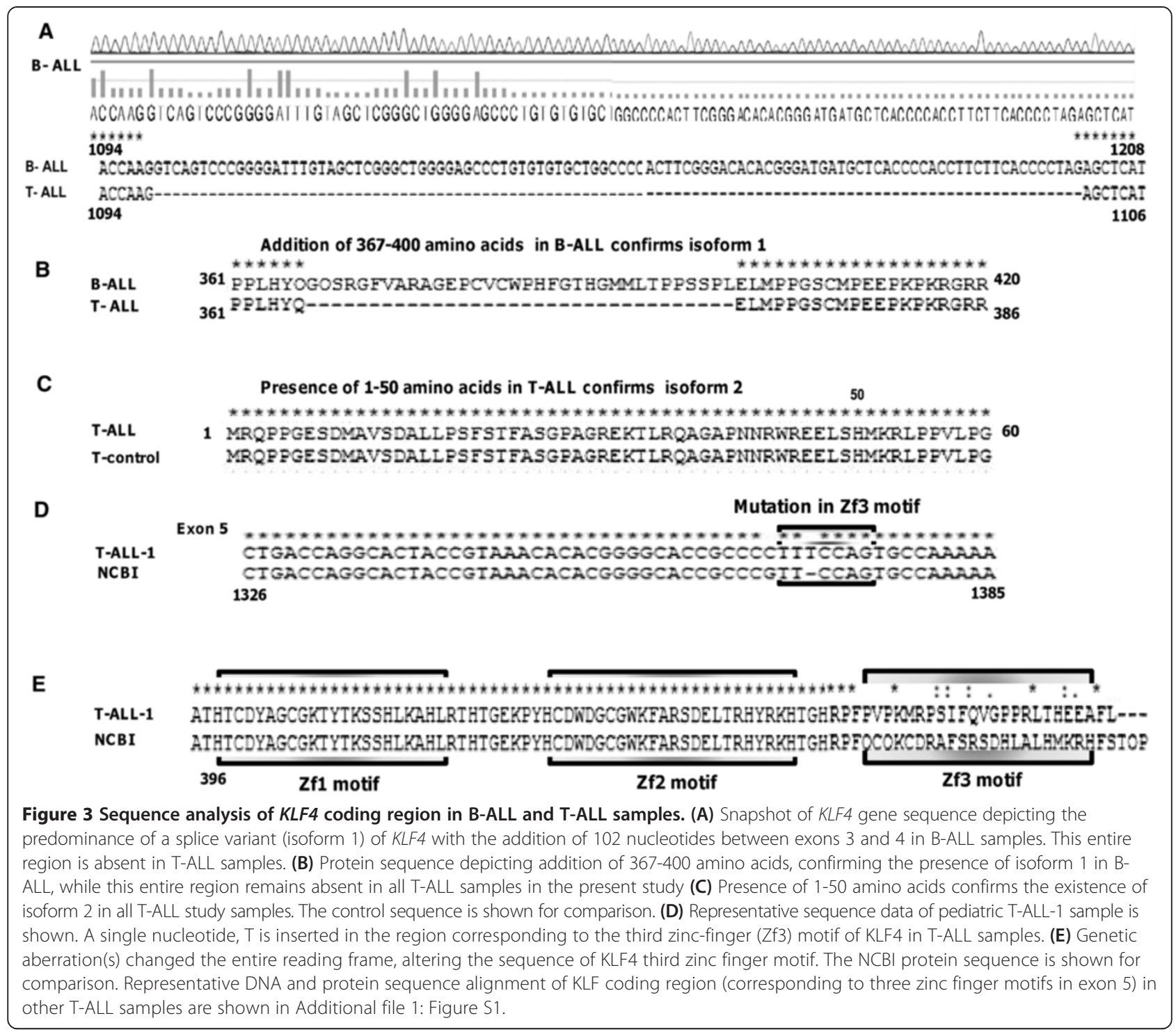

$\left(\mathrm{RNHC}\left(\mathrm{NH}_{2}\right)_{2}^{+}\right)$group of arginine only formed a salt bridge with the anionic carboxylate $\left(\mathrm{RCOO}^{-}\right)$of either glutamic acid or aspartic acid (E446 and R479, E446 and R479, D445-R443). The molecular interactions of modeled wild-type and mutant KLF4 with a 10-bp DNA sequence $\left(5^{\prime}\right.$-cgggcggggc- $\left.3^{\prime}\right)$ in the $P 21^{C I P}$ gene promoter, which is widely recognized as the transcriptional target site for KLF4 protein $[18,19]$, revealed that T-ALL mutant KLF4 was unable to interact with its target DNA sequence as a result of mutation of the $\mathrm{Zf3}$ motif, while wild-type KLF4 exhibited proper cation- $\pi$ and hydrogenbonding interactions with its target DNA sequence (Figure 4E). Docking analysis of wild-type KLF4 revealed that residues Arg458, Lys453, Arg471, and Arg467 displayed cation- $\pi$ interactions with guanine (at positions 11 , 6,7 ) and cytidine 10 (Figure $4 \mathrm{~F}$ and $\mathrm{G}$ ), and residues
His424 and Ser470 formed hydrogen bonds with guanine (at positions 20 and 11), respectively (Figure $4 \mathrm{G}$ and $\mathrm{H}$ ). In contrast, docking of mutant KLF4 with its target DNA sequence exhibited the formation of hydrogen bonds between Arg458 and guanine (at position 3) and cation- $\pi$ interactions between Arg443 and cytidine14 (Figure 4G). The precise details of other non-covalent interactions are discussed in Additional file 3: Figure S3.

Loss of transcriptional activity of mutant KLF4 protein

To confirm the bioinformatics result suggesting that mutant KLF4 was unable to interact with its 10-bp target sequence in the $P 21^{C I P}$ gene promoter in pediatric $\mathrm{T}$ ALL, we transfected a $\beta$-galactosidase $(\beta$-gal) construct under the control of the $P 21^{C I P}$ promoter and harboring a KLF4 site into control and T-lymphoblasts and 


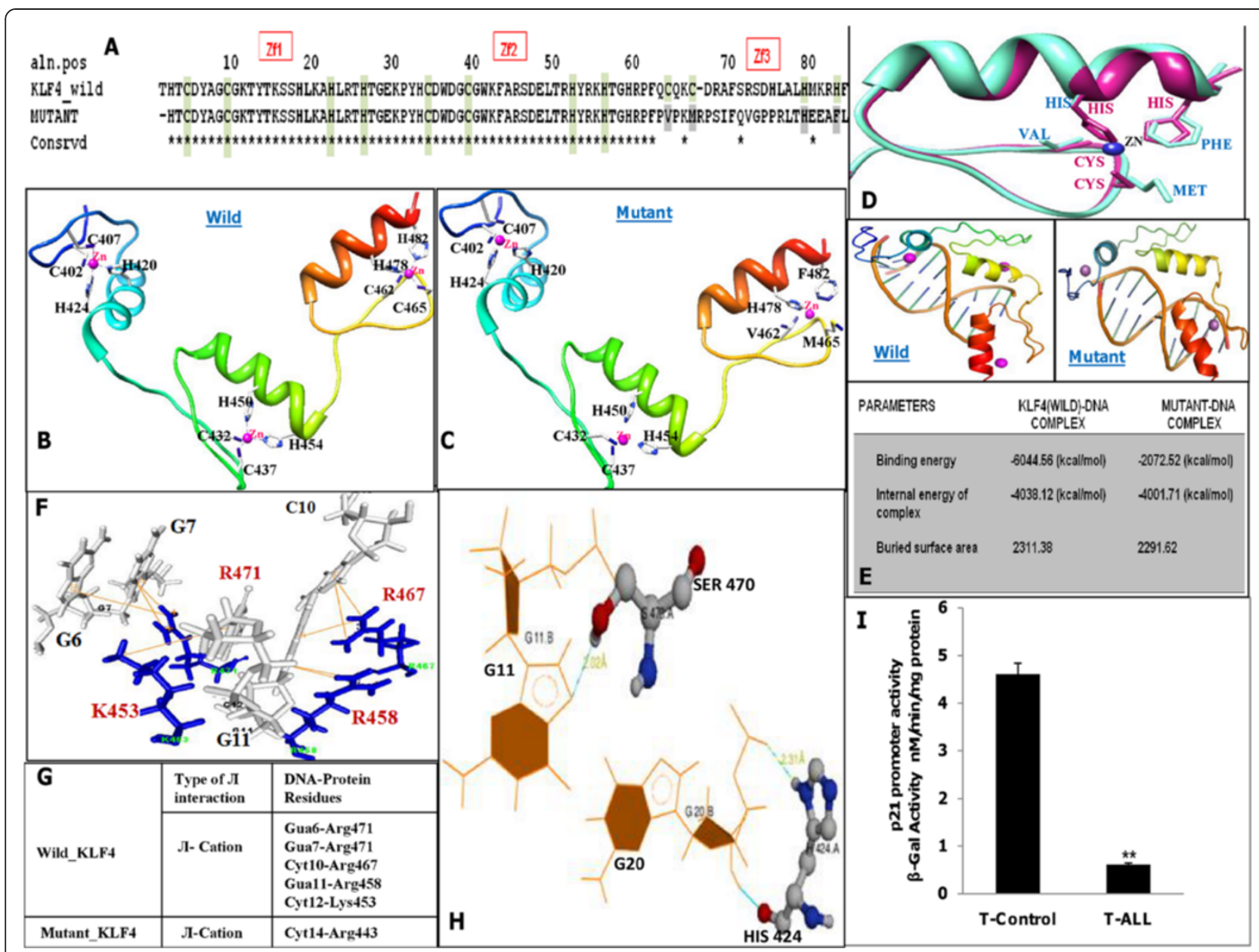

Figure 4 Molecular Modeling and docking studies of wild-type and mutant KLF4. (A) Representative sequence alignment of wild-type KLF4 (derived from control T-cells) with mutant KLF4 (derived from pediatric T-ALL samples). The zinc finger motifs Zf1, Zf2 and Zf3 are highlighted and $\left(^{*}\right)$ indicates conserved residues. The grey region indicates third zinc finger motif (Zf3) with mutated amino acids. (B-C) The structure of zinc finger motifs for wild type (B) and mutant KLF4 (C) were built using MODELLER Program under Accelrys Discovery Studio version 2.5. Zf1; blue, Zf2; green and Zf3; red. Comparison of structural models of the third zinc-finger motif between wild-type and mutant KLF4 revealed the amino acid replacements C462V, C465M, and H482F, which were involved in coordination with zinc (D) Superimposition of Zf3 motif of wild-type (pink) and mutant KLF4 (blue). (E) Molecular interaction of modeled wild-type and mutant KLF4 with its target DNA binding sequence with inserted table depicting the binding energies of the interacting complexes. (F-G) Docking analysis of wild-type KLF4 revealed that residues R458, K453, R471and R467 displayed cation- $\pi$ interactions with guanine (at positions 11, 6, 7) and cytidine 10. In contrast, docking of mutant KLF4 with its target DNA sequence exhibited cation- $\pi$ interactions only between R443 and cytidine 14. (H) Docking analysis of wild-type KLF4 revealed that residues $\mathrm{H} 424$ and $\mathrm{S} 470$ formed hydrogen bonds with guanine (at positions 20 and 11), respectively. Such interactions were missing in mutant KLF4 as a consequence of altered sequence of KLF4 third zinc finger motif; R458 only formed a hydrogen bond with guanine 3. See Additional file 3: Figure S3 (I) $\beta$-galactosidase reporter activity in control and T-ALL lymphoblasts transfected with $\beta$-gal construct. The experiments were repeated thrice and results were reported as relative $\beta$-gal activity. ${ }^{*} \mathrm{P}<0.05$ relative to control.

subsequently incubated for $72 \mathrm{~h}$ at $37^{\circ} \mathrm{C}$ in humidified $5 \% \mathrm{CO}_{2}$ atmosphere. Transfected control cells displayed increased transcriptional activity of the reporter construct containing the $P 21^{C I P}$ promoter compared with T-lymphoblasts (Figure 4I), suggesting that mutant KLF4 protein in T-ALL loses its ability to bind to the KLF4-binding site present in the $P 21^{C I P}$ promoter, and is thus unable to induce $P 21^{C I P}$. In contrast, wild-type KLF4 protein bound to its putative site in the $P 21^{C I P}$ promoter and induced its expression in control T-cells.

\section{Role of SP1 in KLF4-mediated gene expression}

$K L F 4$ has been widely recognized to control cell cycle progression through the induction of $P 21^{C I P}$ and subsequent repression of CCND1 expression [20,21]. It also represses transcription factor $S P 1$ expression, which regulates genes involved in apoptosis $(A A T F)$ and cell proliferation (MYC and BCL3) [22,23]. We therefore investigated the relationship between miR-2909-modulated KLF4 and the genes involved in cellular proliferation and apoptosis. Both B- and T-lymphoblasts derived from 
pediatric ALL patients exhibited significantly lower expression levels of $P 21^{C I P}$ mRNA and protein (Figure 5A and $\mathrm{C}$ ), while mRNA levels of BCL3, MYC and AATF were significantly upregulated (Figure $5 \mathrm{~A}$ and $\mathrm{B}$ ). Previous reports suggest it is likely that the low expression levels of $P 21^{C I P}$ were responsible for the overexpression of CCND1 in both B-ALL and T-ALL (Figure 5D). However, the molecular regulation of KLF4-driven genes such as BCL3, MYC and
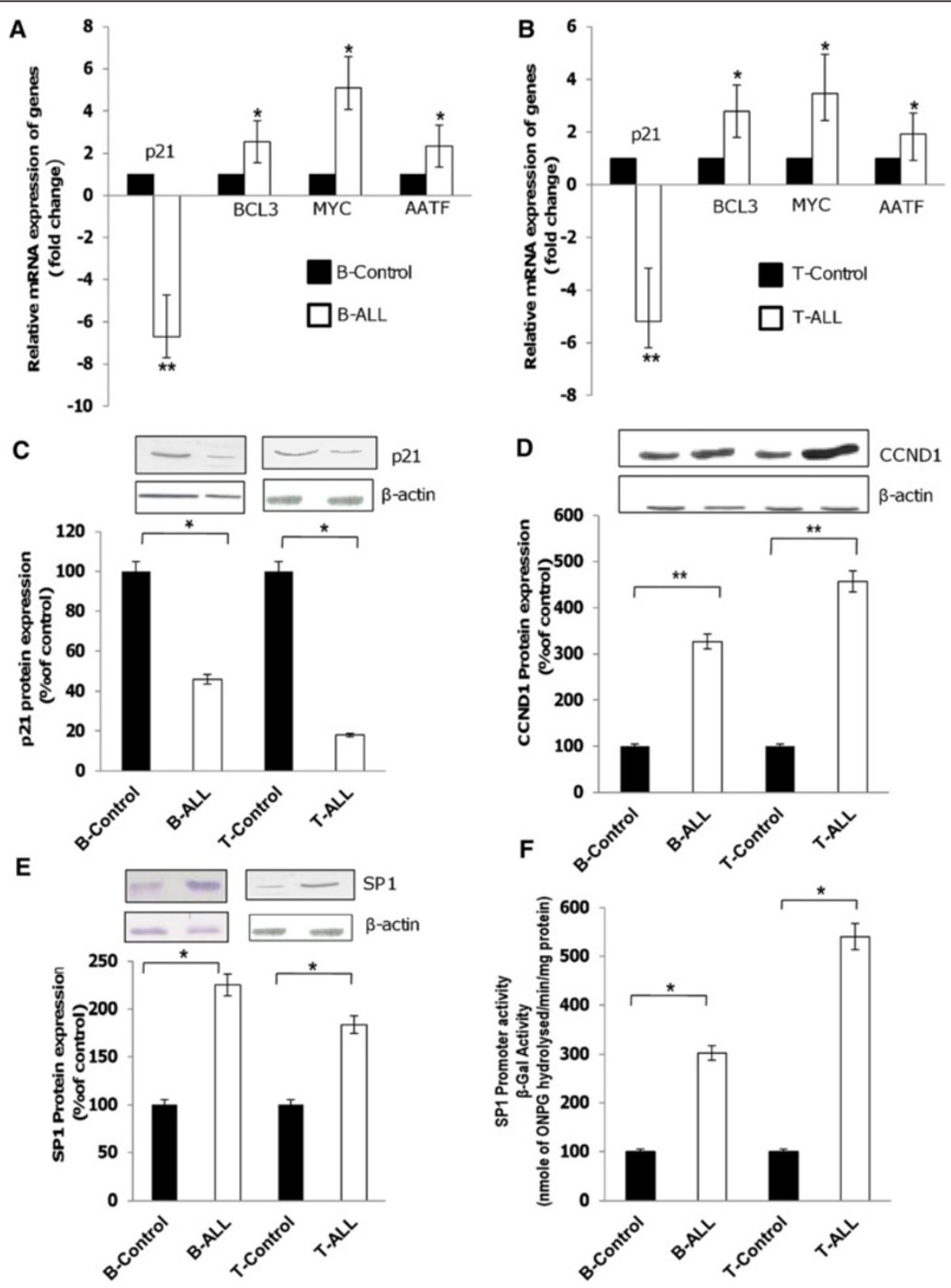

Figure 5 Expression levels of genes involved in cell cycle suppression (KLF4, P21 ${ }^{C I P}$ ), cell proliferation (MYC, CCND1, BCL3, SP1) and apoptosis (AATF) in pediatric acute lymphoblastic leukemias. (A-B) qRT-PCR analysis for expression level of genes including $P 27^{C I P}, B C L 3, M Y C$ and AATF in B-and T-cells from patients with pediatric ALL compared with the corresponding controls. Expression was normalized to $\beta$-actin and each bar represents mean \pm S.D of the experiment performed in triplicate; ${ }^{* *} \mathrm{P}<0.01$; ${ }^{*} \mathrm{P}<0.05$ relative to control $\mathrm{B}$ and $\mathrm{T}$ cells. (C-E) Western blotting to determine protein expression levels of $P 27^{C I P}$ (C), CCND1 (D) and SPI (E) in both B-and T-lineage blasts compared with corresponding controls. Expression was normalized to $\beta$-actin and protein band intensities were determined using Scion Image Analysis Software. Each bar represents mean \pm S.D. of the experiment performed in triplicate; ${ }^{*} \mathrm{P}<0.01 ;{ }^{*} \mathrm{P}<0.05$ relative to control $\mathrm{B}$ and T cells. (F) SP1 transcription factor promoter activity in both B-ALL and T-ALL samples compared with the corresponding controls. B-and T-lymphoblasts were transfected with reporter plasmids containing SP1 response elements, incubated for $72 \mathrm{~h}$ at $37^{\circ} \mathrm{C}$ in humidified $5 \% \mathrm{CO}_{2}$ atmosphere. The experiments were repeated thrice and results were reported as relative $\beta$-gal activity. Each bar represents mean \pm S.D of the experiment performed in triplicate ${ }^{*} P<0.05$ relative to control. 
$A A T F$ remains unclear. Given that the promoter regions of all the above-mentioned genes contain SP1-binding sites (data not shown) and KLF4 has the ability to downregulate SP1 expression, we explored the role of SP1 in this molecular regulation. SP1 protein levels were significantly elevated in both B-ALL and T-ALL samples (Figure 5E). To confirm that the elevated SP1 levels were transcriptionally active, we transfected B- and T-lymphoblasts with reporter plasmids containing SP1 response elements and found that SP1 promoter activity was dramatically increased in both B- and T-lineage blasts compared with corresponding controls (Figure 5F). These results indicate that the reduced expression levels of KLF4 in B-ALL and mutant KLF4 in T-ALL were unable to repress SP1 expression, resulting in increased SP1 levels, which in turn upregulated BCL3, MYC, and AATF expression in both cell types.

miR-2909 regulates cell cycle and apoptosis in B- but not T-lymphoblasts from patients with pediatric ALL

We wanted to determine if the above-mentioned genes were upregulated as a consequence of miR-2909 overexpression in B-ALL. B-lymphoblasts were transfected with
antagomiR-2909 to knockdown miR-2909 expression, resulting in significant upregulation of KLF4 and $P 21^{C I P}$ and downregulation of $M Y C, B C L 3$ and $A A T F$ mRNAs compared with B-lymphoblasts transfected with control scrambled RNA (Figure 6A). KLF4 protein levels were also significantly increased in these cells (Figure 6B). In contrast, antagomiR-2909-transfection of T-lymphoblasts had no significant effect on any of the above genes or on KLF4 protein levels compared with Tlymphoblasts transfected with control scrambled RNA (Figure 6C and D). These results thus revealed that miR2909 plays a key role in the regulation of $P 21^{C I P}, K L F 4$, $A A T F, B C L 3, M Y C$ and CCND1, which control the cell cycle and apoptosis in B-ALL, while miR-2909 loses the capacity to regulate the expression of these genes in $\mathrm{T}$ ALL because they harbor a mutated miR-2909-binding site in the 3'UTR of KLF4 and a mutated KLF4 protein.

We also evaluated the physiological relevance of the miR-2909-regulated genes in the control of the cell cycle and apoptosis in both B-ALL and T-ALL using flow cytometry analysis of antagomiR-2909-transfected cells. The percentage of cells in G1 phase of the cell cycle was dramatically increased, whereas the percentage of cells in $\mathrm{S}$

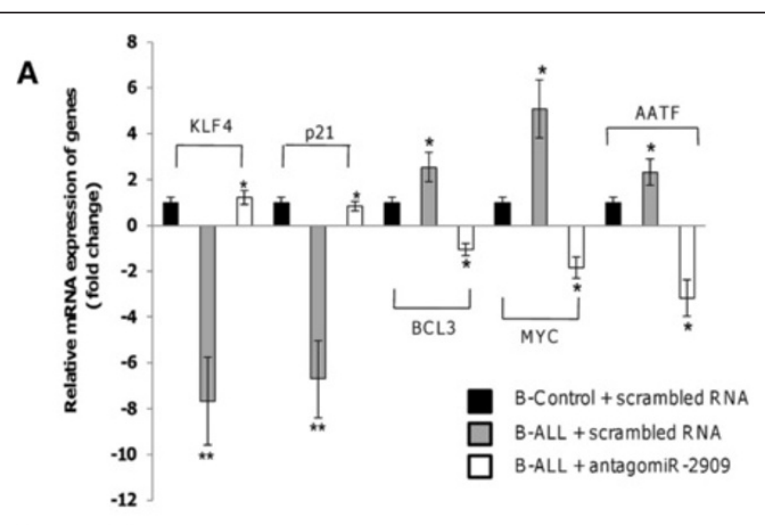

C

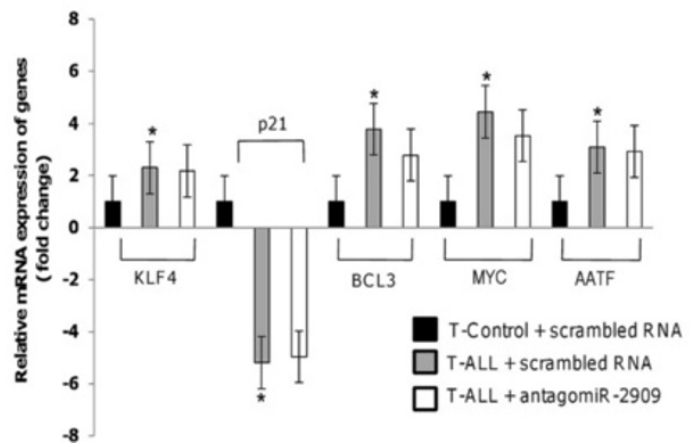

B

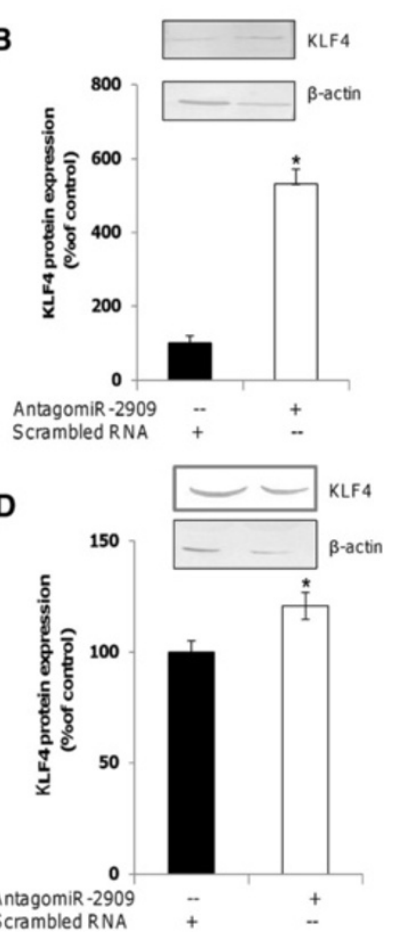

Figure 6 Effect of miR-2909 knockdown on the expression level of various genes in pediatric patients with B-ALL and T-ALL. (A,C). qRT-PCR analysis of genes for KLF4, P21 CIP , BCL3, MYC, AATF in B- (A) and T-lymphoblasts (C) transfected with antagomiR-2909 (50nM) compared with lymphoblasts transfected with control scrambled RNA (50nM). The data representation of mRNA expression levels of the above-mentioned genes in B- and T-lymphoblasts were compared with control B- and T-cells incubated for $48 \mathrm{~h}$ at $37^{\circ} \mathrm{C}$ in humidified $5 \% \mathrm{CO}_{2}$ atmosphere. (B,D) KLF4 protein levels in antagomiR-2909 transfected B- (B) and T-lymphoblasts (D) compared with lymphoblasts transfected with scrambled RNA. Densitometric analysis of protein bands was carried out using Scion Image Analysis Software. Each bar represents mean \pm S.D of the experiment performed in triplicate ${ }^{*} \mathrm{P}<0.05$ relative to control. 
phase was markedly reduced, indicating cell cycle arrest at G1-S phase (Figure 7A) in B-lymphoblasts transfected with antagomiR-2909 compared with control scrambled RNAtransfected B-lymphoblasts. Additionally, antagomiR-2909- transfected B-lymphoblasts showed significantly increased apoptosis as a consequence of downregulation of the $A A T F$ gene (Figure 7B). In contrast, suppression of miR2909 expression in T-lymphoblasts by antagomiR-2909

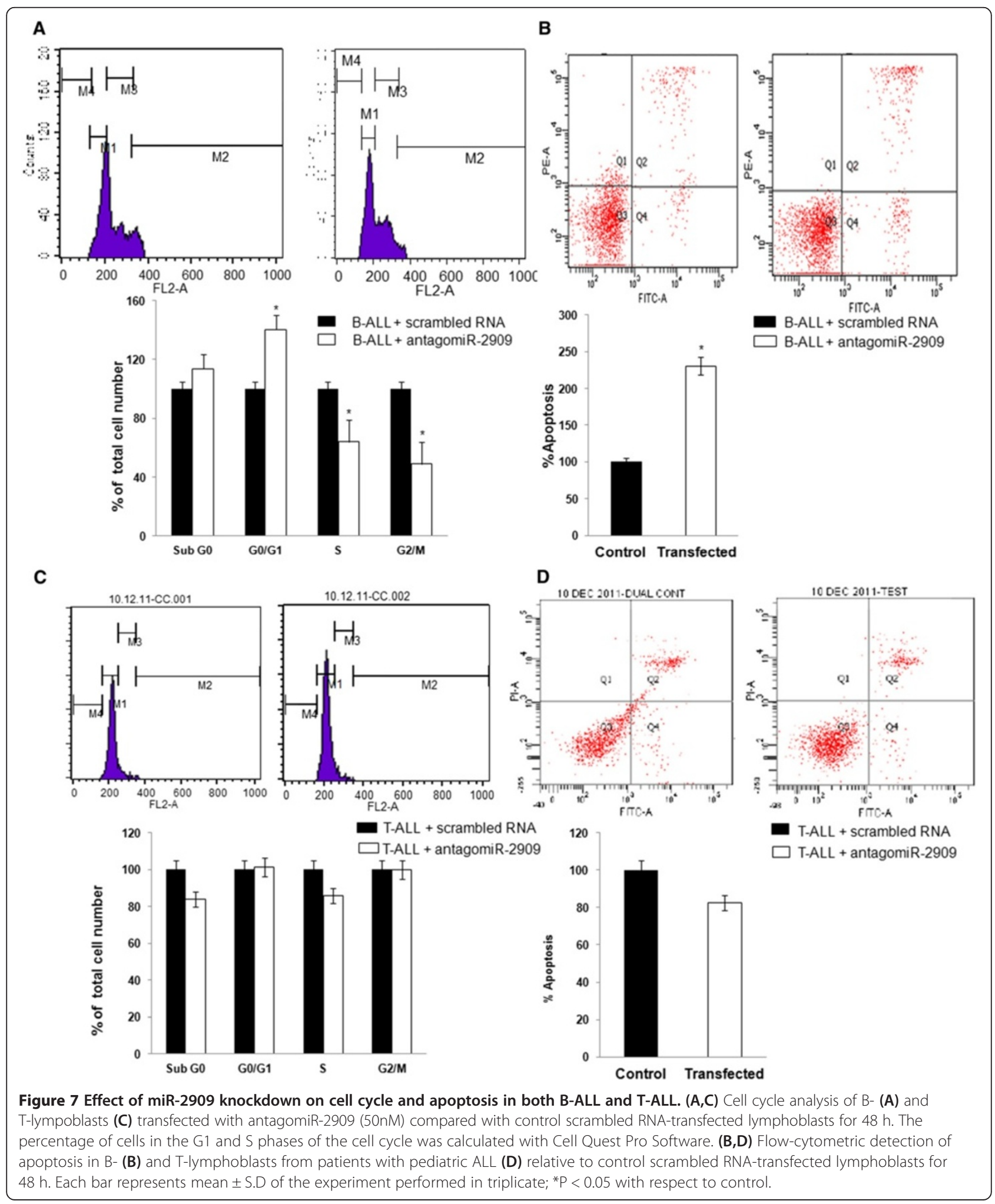




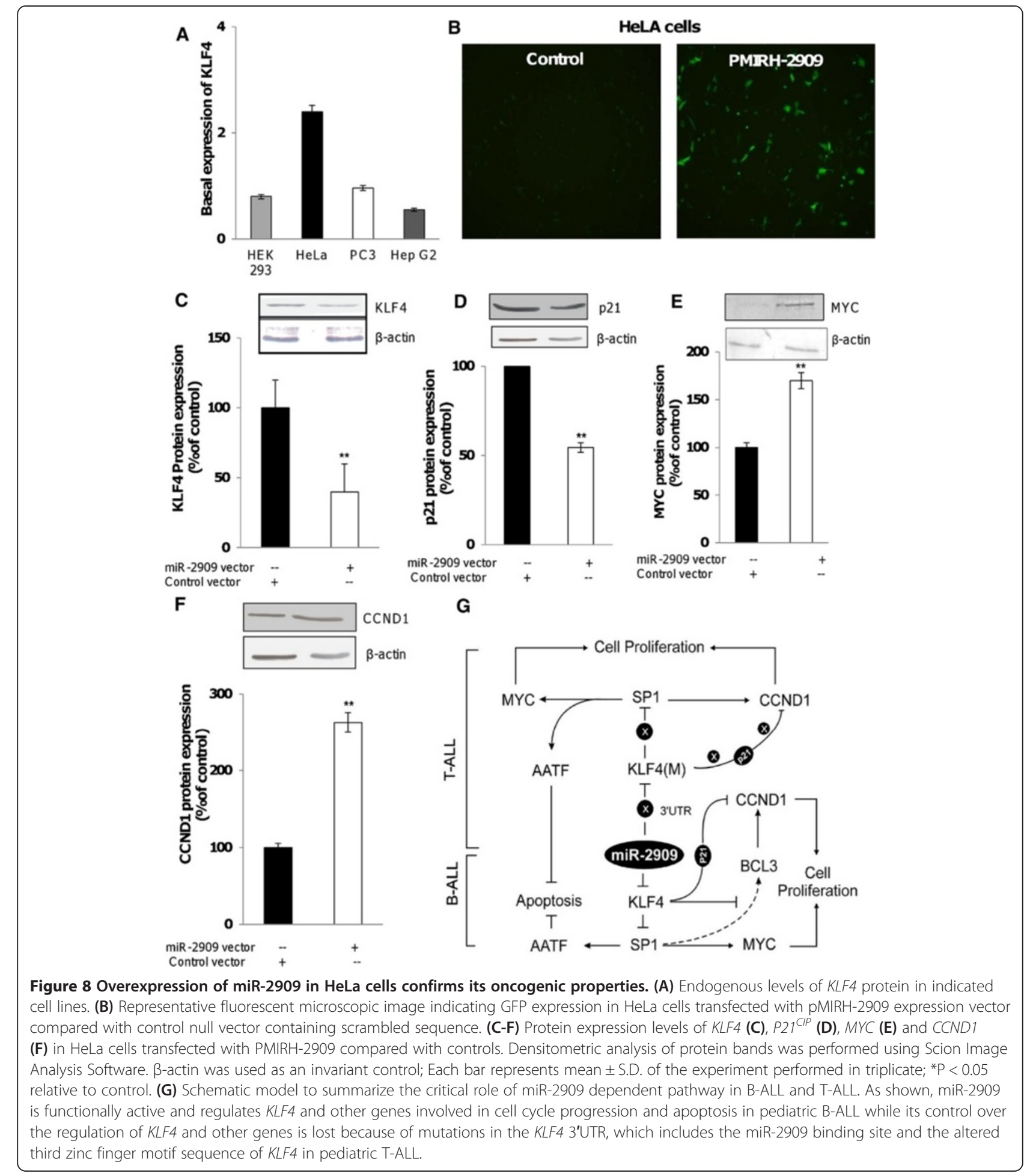

transfection had no effect on the percentage of cells in the G1 and S phases of the cell cycle (Figure 7C) and apoptosis (Figure 7D) relative to T-lymphoblasts transfected with scrambled RNA, further confirming that the mutant KLF4 in T-ALL was non-functional.

\section{Overexpression of miR-2909 in HeLa cells induces the} expression of oncogenes

The elevated levels of miR-2909 in B-ALL reduced expression of the tumor suppressor KLF4, thus signifying its oncogenic potential. To investigate its oncogenic nature 
further, HeLa cells, which express low levels of miR-2909 (Figure 2B) and high levels of KLF4, were transfected with the PMIRH-2909 expression vector to increase miR-2909 expression (Figure 8A and B), and protein expression levels of KLF4, MYC, P21 ${ }^{C I P}$, and CCND1 were analyzed. KLF4 and $P 21^{C I P}$ expression levels were decreased (Figure $8 \mathrm{C}$ and D) and MYC and CCND1 levels were increased (Figure 8E and F) in HeLa cells transfected with PMIRH2909 compared with controls. These results confirm that miR-2909-mediated suppression of KLF4 results in failure of $P 21^{C I P}$ induction and promotion of cell proliferation through upregulated expression of $C C N D 1$ and $M Y C$ proteins.

\section{Discussion}

Mounting evidence has established KLF4 as a transcriptional activator, repressor, tumor suppressor and an oncogene, depending on its genetic context [8]. However, the exact molecular mechanisms whereby KLF4 fulfills these roles remain unknown. Moreover, the pathway regulating KLF4 expression has not been well studied. The present study therefore aimed to investigate the miR-2909-mediated regulation of KLF4 and its downstream functions, especially cell cycle regulation and apoptosis, in both patients with B- and T-ALL. To the best of our knowledge, the results provide the first evidence for two novel mutations in the KLF4 gene in T-ALL: a mutation in the 3'UTR resulted in loss of miR-2909-mediated regulation, and mutation in the Zf1/Zf3 motif rendered $K L F 4$ transcriptionally inactive. In contrast, KLF4 is regulated post-transcriptionally by miR-2909, and suppression of its expression resulted in loss of KLF4 tumor suppressor activity in pediatric B-ALL.

Previous studies involving a variety of epithelial tumors have shown that the expression of the zinc-finger transcription factor KLF4 is silenced by promoter hypermethylation [9]. KLF4 is also known to be inactivated by methylation in adult T-cell leukemia [24]. Recent studies have revealed direct correlations between altered miRNA levels and progression of hematological malignancies, such as the effects of downregulation of miR-15a and miR-16-1 in chronic lymphocytic leukemia [13] and amplification of miR-155 in B-cell lymphoma, Hodgkin's lymphoma [25], Burkitt's lymphoma [26] and in human breast cancer cell lines [27]. However, the role of miRNAs in the pathogeneses of pediatric B- and T-cell ALL remains unknown. Earlier studies from our laboratory reported that the AATF gene encodes miR-2909, the functional importance of which remains unexplored in terms of the pathogeneses of B- and T-cell ALLs. We therefore investigated the expression of miR-2909 and its regulation of its target gene KLF4. miR-2909 levels were significantly elevated in both B- and T-ALL compared with corresponding age-matched control subjects. Moreover, we found a reciprocal relationship between miR-2909 and KLF4 expression in B-ALL, but not in T-ALL, which displayed significantly higher levels of KLF4 expression despite elevated levels of miR-2909. The observed low levels of KLF4 in B-ALL compared with healthy subjects were in accordance with a previous report [9].

We subsequently explored the possibility that patients with T-ALL may harbor mutations in the KLF4 3'UTR, which includes the miR-2909 binding site. Sequence analysis of the 3'UTR revealed altered nucleotides in the 'seed region', which is important for target specificity, in T-ALL but not in B-ALL patients. We further demonstrated that miR-2909 targeted the KLF4 3'UTR isolated from B-ALL patients, resulting in repression of KLF4 protein, but not that isolated from T-ALL patients with the mutated miR-2909 binding site, leading to increased levels of KLF4 protein. Using an miRNASelect pMIRGFP reporter assay, we confirmed that miR-2909 targeted KLF4 in B-ALL but not in T-ALL by constructing plasmids containing the 3'UTR and the miR-2909 target site from the B-cell (pGFP-KLF4-3'UTR-B) and T-cell lineages (pGFP-KLF4-3'UTR-T). Transfection of the pGFP-KLF4-3'UTR-B plasmid into HEK 293 cells resulted in a $52.07 \%$ reduction in GFP expression, whereas cells expressing pGFP-KLF4-3'UTR-T showed no noticeable difference compared with cells transfected with pGFP plasmid without the 3'UTR insert. These results clearly revealed the ability of miR-2909 to repress KLF4 expression in pediatric ALL B-cell, but not T-cell lineages. Our results thus highlight the molecular differences in miR2909-mediated differential regulation of KLF4 between B- and T-ALL. Importantly, our results identifying KLF4 as a target of miR-2909 in B-ALL are similar to published reports of miRNA-145-mediated repression of KLF4 in human embryonic stem cells [28] and miR$10 \mathrm{~b}$ regulation of KLF4 in human esophageal cancer cell lines [29]. However, these results in pediatric T-ALL provide the first functional demonstration of loss of repression of any target gene as a result of a mutated seed sequence for any miRNA in any disease.

We also explored why elevated KLF4 levels failed to control excessive proliferation in T-ALL patients by amplifying the full-length KLF4 sequences from B- and T-ALL samples. Most patients with B-ALL expressed KLF4 isoform 1, while T-ALL patients expressed isoform 2. However, the precise contributions of each isoform to the overall functions of KLF4 are not well understood and it will be interesting to validate these findings in a larger cohort to improve our understanding of its implications for the pathogeneses of ALL. In addition to identifying the isoforms present in B- and T-ALL, we also discovered nucleotide insertions or deletions in the Zf1 or Zf3 motif in pediatric T-ALL samples, resulting in frameshift mutations and complete alteration of the 
zinc-finger motif sequence, which could possibly destroy its DNA-binding affinity.

The crystal structure of the zinc-finger domain of KLF4 bound to target DNA was recently reported [30], and we exploited this structural information to construct homology models and perform docking studies of wildtype and mutant KLF4 with the 10-bp KLF4 target DNA sequence $\left(5^{\prime}\right.$-cgggcggggc- $\left.3^{\prime}\right)$. The modeled structure of the Zf3 motif in the mutant KLF4 revealed the replacement of three key residues (C462V, C465M and H482F) involved in coordination with zinc. Moreover, the docking results demonstrated that mutant KLF4 was unable to interact with its 10 -bp target sequence in the $p 21^{C I P}$ gene promoter, while wild-type KLF4 displayed cation- $\pi$ and hydrogen-bonding interactions with its target sequence in controls. These results clearly demonstrate the importance of the Zf3 motif in KLF4-mediated functional regulation of its target gene. These findings are consistent with a previous study demonstrating that KLF4-mediated macrophage differentiation was primarily controlled by the two C-terminal Zf2 and Zf3 motifs, while Zf1 imparted little specificity [30]. More importantly, we provided experimental proof to support the docking studies, showing that mutant KLF4 in Tlymphoblasts was unable to induce $p 21^{C I P}$ promoter activity, while $K L F 4$ in control T-cells increased $p 21^{C I P}$ promoter reporter activity. A similar phenomenon was reported in colorectal cancer cell lines, where KLF4 mutations resulted in reduced $p 21^{C I P}$ promoter activity [31].

KLF4 acts as cell cycle regulator and functions as a tumor suppressor through its ability to induce p $21^{\mathrm{CIP}}$ [20] and suppress SP1 expression [22]. Based on these findings, we studied the expression levels of genes involved in this process in ALL patients. We observed significant reductions in $p 21^{C I P}$ and increases in SP1 in B-ALL as a result of suppressed KLF4 expression. In contrast, elevated levels of mutant KLF4 failed to induce $p 21^{C I P}$ or inhibit SP1 expression in T-ALL, leading to low levels of $p 21^{C I P}$ and high levels of $S P 1$, respectively. Moreover, the increased levels of SP1 were associated with increased transcriptional activity of a reporter construct containing an SP1 response element in both Band T-ALL subtypes. Earlier work from our laboratory showed that SP1 upregulated AATF and MYC gene in Jurkat cells [23]. Additionally, bioinformatics analysis revealed the presence of an $S P 1$ response element in the promoter regions of AATF, MYC and BCL3 (data not shown). Consistent with these results, we also confirmed that upregulated $S P 1$ levels resulted in increased BCL3, $A A T F$ and $M Y C$ expression in both B- and T-ALL subtypes. Our results therefore suggest that KLF4-mediated regulation of these genes most likely occurs through $S P 1$ in both B- and T-ALL. The current study also assessed the key role of miR-2909 in the regulation of the above- mentioned genes. Results in B-lymphoblasts transfected with antagomiR-2909 confirmed that miR-2909 regulates these genes through KLF4. Transfection of pediatric Blymphoblasts with antagomiR-2909 for $48 \mathrm{~h}$ resulted in significant upregulation of KLF4 expression. Furthermore, antagomiR-2909-transfected B-lymphoblasts revealed significant repression of the $A A T F, M Y C$ and $B C L 3$ genes. In contrast, antagomiR-2909-transfection had no such effects in pediatric T-lymphoblasts, as a result of failure of miR-2909 to bind to the mutated 3' UTR of KLF4 and mutated KLF4 protein. We subsequently tested the functional relevance of increased KLF4 in antagomiR-2909-transfected B- and T-lymphoblasts. B-lymphoblasts showed cell cycle arrest at G1/S phase, most likely through increased expression of $p 21^{C I P}$, and increased apoptosis, possibly resulting from $A A T F$ suppression. However, no such changes were seen in antagomiR-2909-transfected pediatric T-lymphoblasts, despite increased levels of $K L F 4$, which was rendered transcriptionally inactive as a result of the altered $\mathrm{Zf3}$ motif, which lacked DNA-binding activity. In addition, antagomiR-2909-transfected B-lymphoblasts also exhibited decreased mRNA expression of $M Y C$ and $B C L 3$ mRNA expression levels, which may reduce cell proliferation. These results were similar to other reported studies showing increased expression of $p 21^{C I P}$ and decreased expression of $M Y C$ and cyclin D2 in pro/pre-B cells expressing KLF4 [32]. Furthermore, miR-2909 overexpression and its ability to target the tumor suppressor KLF4 suggest that it displays oncogenic properties in B-ALL. To prove this, we overexpressed miR-2909 in HeLa cells to silence KLF4 expression. This resulted in significantly increased CCND1 and $M Y C$ and decreased $\mathrm{p} 21^{\mathrm{CIP}}$ protein levels. These results are consistent with previous findings, which showed that KLF4-silencing in HeLa cells promoted cell growth and tumor formation [33].

Collectively, our experimental results suggest the existence of a molecular pathway as depicted in Figure 8G, which summarizes the differential nature of the miR2909-KLF4 axis in B-ALL and T-ALL. The proposed pathway explains how high mutant KLF4 protein expression in T-ALL subjects is unable to induce $p 21^{C I P}$, which plays a major role in inhibiting CCND1 expression. $\mathrm{Mu}-$ tant KLF4 protein also failed to repress SP1 expression, a gene known to be downregulated by $K L F 4$, in parallel with upregulation of the oncogenes $M Y C, B C L 3$ and the anti-apoptotic $A A T F$, potentially leading to unbridled Tcell transformation. A similar phenomenon is proposed in B-ALL subjects, wherein downregulation of KLF4 protein by miR-2909 overexpression results in B-cell transformation. Significantly, our studies revealed two breakpoints in the miR-2909-KLF4 axis caused by mutation in the 3'UTR-KLF4 and the altered Zf3 motif sequence, leading to loss of miR-2909 binding to the 
KLF4 3'UTR and loss of KLF4 binding to DNA sequences in target genes in T-cell ALL subjects. Moreover, structural modeling of the zinc-finger motif of KLF4 uncovered the precise molecular basis of loss of DNA binding in T-ALL, associated with alterations of three key amino acid residues involved in zinc coordination. Overall, our findings suggest that KLF4 function is compromised in T-cells derived from patients with pediatric T-ALL because of alteration of the $\mathrm{Zf} 3$ motif sequence as a result of a frameshift mutation. Furthermore, its regulation by miR-2909 is impaired because of a mutation in the miR-2909 target sequence present in the 3'UTR of the KLF4 mRNA. In B-ALL subjects, KLF4 function is compromised because of miR-2909dependent downregulation. KLF4 is therefore unable to act as a tumor suppressor gene in either pediatric B-ALL or T-ALL. These results raise the possibility of the existence of similar KLF4 mutations in other tissues. However it was not possible to investigate the presence of such mutations in other tissues from the study patients because of ethical considerations.

\section{Conclusions}

The present study discovered that elevated levels of the novel miR-2909 target the tumor suppressor KLF4, which regulates the cell cycle and apoptosis in pediatric ALL. miR-2909-mediated downregulation resulted in loss of KLF4 activity in B-ALL. In contrast, miR-2909 failed to regulate KLF4 expression in T-ALL because of mutations in the KLF4 3'UTR, which includes the miR2909 binding site. The molecular defect responsible for loss of KLF4 function in T-ALL despite its elevated level, lies within the Zf3 motif, which is altered as a result of a frameshift mutation. Homology modeling/docking studies and $p 21^{C I P}$ promoter activity confirmed the lack of functional activity of mutant KLF4. Comprehensive sequence analysis of KLF4 identified the predominance of isoform 1 in most patients with pediatric B-ALL, and of isoform 2 in patients with T-ALL. These results demonstrate the existence of a novel miR-2909-KLF4 molecular axis able to differentiate between B- and T-ALL pathogeneses, and which may provide a new diagnostic/prognostic marker to evaluate the pathogenesis of ALL in pediatric subjects. Our results also suggest that screening for these two mutations could be developed as a diagnostic strategy to differentiate between these two ALL subtypes. Overall, this study provides the basis for further research that could lead to a novel therapeutic approach for patients with B-and T-cell ALL.

\section{Materials and methods Materials}

The reagents were procured as follows: MiniMACS ${ }^{\mathrm{Tm}}$ Starting Kit (Miltenyi Biotech, Auburn, CA); miRNeasy mini kit, miScript Reverse transcription kit, miScript SYBR Green kit, Universal primer for amplifying miR2909, Pfu polymerase (Fermentas, Vilnius, Lithuania); Qiaquick PCR purification kit (Qiagen, Valencia, CA); pBlue TOPO reporter vector, Lipofectamine 2000 and $\beta$ Gal Assay Kit (Invitrogen,Carlsbad, USA); anti-KLF4 (Abcam), anti-p21 ${ }^{\mathrm{CIP}}$, anti-SP1, anti-MYC, anti-CCND1, anti- $\beta$ actin; Annexin V-FITC apoptosis kit (SigmaAldrich, St.Louis, MO, USA); pMIR-GFP Reporter Vector (Cell Biolabs, San Diego, CA, USA); miRCURY LNA ${ }^{\mathrm{m}}$ miR-2909 power inhibitor (EXIQON, Denmark); PMIRH2909 lentiviral construct (System Biosciences, CA, USA).

\section{Patient samples}

The pediatric B-ALL, T-ALL samples and age-matched control samples were obtained from Advanced Pediatric Centre, Post Graduate Institute of Medical Education and Research (PGIMER), Chandigarh, India with prior consent from their guardian through ethical approval by the Institutional Review Board of PGIMER. Total samples of B-ALL and T-ALL were 30 and 20 respectively. Age-matched subjects $(n=50)$ with no manifestations of any haematological malignancy were treated as control. All patients were $<14$ years of age and flow cytometric immunophenotyping showed mostly cells in the blast/ progenitor region (SSC low to moderate, CD45 negative to $\operatorname{dim}$ ) comprising $\sim 80 \%$ of all singlet events acquired. Patients undergoing chemotherapy and cases with mixed phenotype acute leukemia (MPAL) and relapsed cases from pediatric acute lymphoblastic leukaemia (ALL) were excluded in the present study. Diagnosis of pediatric ALL samples into B- and T-cell lineage was evaluated by immunophenotyping using CD19, CD22, CD79a, CD10, CD20, and CD24 for B-ALL and CD7,CD2,CD3,CD5 for T-ALL. Mononuclear cells were isolated using FicollHypaque density gradient method [34]. B- and T-cells were purified using MiniMACS ${ }^{\text {тм }}$ Separator Kit.

\section{RNA Extraction, cDNA synthesis and qRT-PCR}

Total RNA including the small RNA was extracted from patient samples using miRNeasy mini kit in accordance with the manufacturer's instructions. The quality and quantity of extracted RNAs were analyzed using electrophoresis and optical density measurement at $260 \mathrm{~nm}$; cDNA synthesis was performed via miScript Reverse transcription kit as per suppliers's instructions. For assaying gene expression, miScript SYBR Green Mix and the Realtime PCR (Stratagene, San Diego, CA, USA) were used. The qRT-PCR reaction was performed with a starting temperature of $95^{\circ} \mathrm{C}$ for $10 \mathrm{~min}$, followed by 35 cycles of $45 \mathrm{~s}$ at $94^{\circ} \mathrm{C}, 30 \mathrm{~s}$ at $56^{\circ} \mathrm{C}$, and $45 \mathrm{~s}$ at $72^{\circ} \mathrm{C}$. The small non-coding nuclear RNA U6 and $\beta$-actin were used as an invariant controls for normalizing the expression of miR-2909 and other genes respectively. The 2- ${ }^{\Delta \Delta C T}$ 
method was used to calculate the relative expression of target genes.

\section{Immunoblotting}

Total cellular protein was extracted using Laemmli's buffer [35] and the protein levels of KLF4, $221^{C I P}, S P 1, M Y C$ and $C C N D 1$ was determined through western blotting using appropriate antibodies as described previously [36]. $\beta$-actin antibody was used as an internal control. Scion Image Analysis software was used for densitometry analysis and the results were expressed as intensity ratio of target protein to $\beta$-actin protein taken as arbitrary unit.

\section{DNA sequencing}

Primer sets were designed to amplify the full coding region and 3'untranslated region of KLF4 in ALL samples using $P f u$ polymerase. The resultant PCR products were purified using Qiaquick PCR purification kit and sequenced to detect the presence of any genetic aberration (s) in KLF4 in samples from pediatric patients with ALL. The sequence data was analysed using Cluster X 2.0.12 Software (http://www.clustal.org/clustal2) [37].

\section{Plasmid constructs and reporter assays}

Full length 3'UTR of KLF4 in B-ALL was cloned into miRNASelect ${ }^{\text {tw }}$ pMIR GFP reporter vector; designated as pGFP-KLF4-3'UTR-B which carried no substitution of nucleotides within miR-2909 target site in KLF4 3'UTR. Mutant 3'UTR of KLF4 present in T-ALL was named as pGFP-KLF4-3'UTR-T with substitution of nucleotides within core binding site in KLF4 3'UTR. The plasmid constructs were transfected in HEK-293 cells. After $48 \mathrm{~h}$, fluoresence microscopy and FACS analysis was performed to quantitate the number of cells expressing GFP. For $p 21^{C I P}$ promoter analysis, promoter sequence of $p 21^{C I P}$ with putative KLF4 binding site was cloned into pBlue TOPO reporter vector with subsequent transfection of $\beta$-gal construct into control and T- lymphoblasts. For analysis of SP1 transcriptional activity, $\mathrm{B}$ - and T-lymphoblasts with reporter plasmids containing $S P 1$ response elements were transfected. $\beta$ galactosidase activity was measured $72 \mathrm{~h}$ after transfection. To knockdown miR-2909 expression, leukemia cells were transfected with miRCURY LNA ${ }^{\text {tix }}$ miR-2909 power inhibitor. To increase miR-2909 expression, HeLa cells were transfected with the PMIRH-2909 expression vector. All the transfections were performed with Lipofectamine 2000 transfection reagent according to the manufacturer's instructions.

\section{Cell cycle analysis and apoptosis assays by Flow cytometry}

Cell cycle analysis and apoptotic assays was done on leukemia cells transfected with antagomiR-2909 (50 nM) and scrambled RNA (50 $\mathrm{nM}$ ) for $48 \mathrm{~h}$ in RPMI 1640 medium supplemented with $10 \% \mathrm{FBS}, 100 \mathrm{U} / \mathrm{ml}$ penicillin and $100 \mu \mathrm{g} / \mathrm{ml}$ streptomycin under $5 \% \mathrm{CO}_{2}$ at $37^{\circ} \mathrm{C}$. For cell cycle experiments, cells were fixed in $70 \%$ ethanol and stained with PI. Cells percentage at different phases were analysed with FACSCalibur cytometer and Cell Quest Pro software (Becton Dickinson, NJ, USA). For apoptosis assays, cells were stained with FITC Annexin V coupled with propidium iodide and apoptosis was measured using BD FACS Diva Software (Becton Dickinson, FACS Canto II).

\section{KLF4 structural model \& docking with target DNA}

The structural models of zinc finger motifs of wild-type and mutant KLF4 were modeled with template PDB ID: 2WBUA. The Homology models were built using MODELLER (9.9) [38]. Model validation was performed using Verify-3D (http://nihserver.mbi.ucla.edu/Verify_3D/) [39] and PROCHECK [40]. The quality of the final models was evaluated from Ramachandran plot (Additional file 3: Figure S3B). Molecular visualization and structural alignment was done using CHIMERA http://bioinformatics. org/wiki/Chimera [41] and PYMOL http://www.pymol. org/ [42]. Target DNA sequence (5'-cgggcggggc-3') in $p 21^{C I P}$ promoter was modeled into B-FORM using DNA analysis servers [43]. Docking studies were performed using High Ambiguity-Driven bimolecular Docking (HADDOCK) under solvated conditions $[44,45]$. Cation- $\pi$ interactions were analysed with CAPTURE http://capture. caltech.edu/ [46].

\section{Statistical analysis}

Statistical analyses were performed by SPSS Windows version 19. Data was expressed as mean \pm S.D of the experiments performed in triplicate. Student's $t$ test or MannWhitney-Wilcoxon test was performed to determine the significance of difference between two groups. Differences were considered significant at $\mathrm{p}<0.01$ and $\mathrm{p}<0.05$.

\section{Additional files}

\footnotetext{
Additional file 1: Figure S1. Sequence analysis of KLF4 coding region in pediatric T-ALL samples. (A-F) Representative DNA sequence alignment of KLF4 coding region (corresponding to the three zinc finger motifs in exon 5) in T-ALL samples. NCBI sequence is shown for comparison. Sequence analyses indicate insertion (A), deletion (B-F) of nucleotides in the first or third zinc finger motif ( $Z f 1, Z f 3)$ of KLF4 in T-ALL samples. These genetic aberration(s) changed the entire reading frame, altering the sequence of KLF4 third zinc finger motif and potentially destroying its DNA-binding affinity (G) Protein sequence alignment of these same $6 \mathrm{~T}$-ALL samples with respect to NCBI. Identical Zf1 and Zf2 motif in pediatric T-ALL samples is highlighted.

Additional file 2: Figure S2. Sequence analysis of KLF4 coding region in pediatric B-ALL samples. (A,B) Representative DNA (A) and protein sequence alignment $(\mathbf{B})$ of KLF4 coding region (corresponding to the three zinc finger motifs; Zf1, Zf2, Zf3 in exon 5) in all B-ALL samples in the present study. NCBI sequence is shown for comparison.
} 
Sequence analysis revealed no genetic aberrations in any of the three zinc-fingers regions of KLF4 in samples from pediatric patients with B-ALL, suggesting that the conformation of KLF4 was unaffected in these patients (sample size 10).

Additional file 3: Figure S3. Structural models and docking studies of wild-type and mutant KLF4 (A) Predicted residues in the active site of KLF4 were Arg449, Arg467, Lys453, Gly456, His457, Arg458, Ser470, Arg471 and His474 as given by CASTP server. Most of the active residues reside in the second and third zinc finger motifs; Zf2 and Zf 3 as indicated by green dots. (B) Ramachandran plot of wild-type and mutant KLF4 which was built using PROCHECK; wild- type KLF4 has $82.7 \%$ of residues in favoured region and the remaining $16.0 \%$ in additionally allowed regions; mutant KLF4 has $81.4 \%$ residues in the most favoured region and remaining $17.1 \%$ in additionally allowed regions. (C-D) Representative table exhibited the formation of hydrogen bonds between Ser 470 and guanine (at position 11) in wild-type (C) and between Arg458 and guanine (at position 3) in mutant (D) KLF4 (E-F) Representative table showed noncovalent interactions, primarily electrostatic hydrophobic and Van der waals forces between protein residues and bases (quanine and cytidine) in wildtype (E) and mutant KLF4 (F). DNA bases within the parentheses interact simultaneously with its corresponding protein residue. All the active site residues in wild-type KLF4 displayed hydrophobic and Van der waal interactions, in contrast only few active site residues in mutant KLF4 were involved in these interactions.

\section{Abbreviations}

ALL: Acute lymphoblastic leukemia; miR: microRNA; B-ALL: B-cell lineage acute lymphoblastic leukemia; T-ALL: T-cell lineage acute lymphoblastic leukemia; AATF: Anti-apoptotic transcription factor; KLF4: Kruppel like factor transcription factor 4; BCL3: B-cell lymphoma 3-encoded protein; CCND1: Cyclin D1; Zf1: First zinc finger motif; Zf2: Second zinc finger motif; Zf3: Third zinc finger motif; SP1: Specificity protein transcription factor-1; UTR: Untranslated region; CD: Cluster of differentiation; GFP: Green fluorescent protein; $\beta$-gal: $\beta$-galactosidase.

\section{Competing interests}

The authors have no competing interests.

\section{Authors' contributions}

The work was conceived by DK and executed by DM and NC. Patients were supplied by RK. All authors read and approved the final manuscript.

\section{Acknowledgements}

We acknowledge Dr. Neelam Varma for her support in the diagnosis of leukemic samples through Immunophenotyping. We thank Dr. Nitin Patel for his invaluable inputs and critical reading of the manuscript. The study was supported by the funds provided by Indian Council of Medical Research (ICMR), New Delhi (India). http://www.icmr.nic.in/ and this funding agency had no role in designing the experiment, sample collections and analysis done or preparation of this manuscript.

\section{Author details}

${ }^{1}$ Department of Experimental Medicine \& Biotechnology, Postgraduate Institute of Medical Education \& Research, Chandigarh, India. ${ }^{2}$ Department of Advanced Pediatrics Centre, Postgraduate Institute of Medical Education \& Research, Chandigarh, India. ${ }^{3}$ Department of Experimental Medicine \& Biotechnology, Research Block B, Postgraduate Institute of Medical Education \& Research, Chandigarh 160012, India.

Received: 8 October 2013 Accepted: 10 July 2014 Published: 18 July 2014

\section{References}

1. Pieters $R$, Carroll WL: Biology and treatment of acute lymphoblastic leukemia. Pediatr Clin North Am 2008, 55:1-20.

2. Armstrong SA, Look AT: Molecular genetics of acute lymphoblastic leukemia. J Clin Oncol 2005, 23:6306-6315.

3. Passananti C, Floridi A, Fanciulli M: Che-1/AATF, a multivalent adaptor connecting transcriptional regulation, checkpoint control, and apoptosis. Biochem Cell Biol 2007, 85:477-483.
4. Kaul D, Hussain A: Cellular AATF gene encodes a novel miRNA that can contribute to HIV-1 latency. Indian J Biochem Biophys 2009, 46:237-240.

5. Kaul D, Sasikala M, Raina A: Regulatory role of miR-2909 in cell-mediated immune response. Cell Biochem Funct 2012, 30:500-504.

6. Sharma M, Sharma S, Arora M, Kaul D: Regulation of cellular CCND1 gene by arsenic is mediated through miR-2909. Gene 2013, 522:60-64.

7. McConnell BB, Yang WV: Mammalian Kruppel-like factors in Health and Diseases. Physiol Rev 2010, 90:1337-1381.

8. Rowland BD, Peeper DS: KLF4, p21 and context-dependent opposing forces in cancer. Nat Rev Cancer 2006, 6:11-23.

9. Guan H, Xie L, Leithäuser F, Flossbach L, Möller P, Wirth T, Ushmorov A: KLF4 is a tumor suppressor in B-cell non-Hodgkin lymphoma and in classic Hodgkin lymphoma. Blood 2010, 116:1469-1478.

10. Lutherborrow M, Bryant A, Jayaswal V, Agapiou D, Palma C, Yang YH, Ma DDF: Expression profiling of cytogenetically normal acute myeloid leukemia identifies MicroRNAs that target genes involved in monocytic differentiation. Am J Hematol 2011, 86:2-11.

11. Schotte D, Pieters R, Den Boer ML: MicroRNAs in acute leukemia: from biological players to clinical contributors. Leukemia 2012, 26:1-12.

12. Ambros $V$ : The functions of animal microRNAs. Nature 2004, 431:350-355.

13. Calin GA, Dumitru CD, Shimizu M, Bichi R, Zupo S, Noch E, Aldler H, Rattan S, Keating M, Rai K, Rassenti L, Kipps T, Negrini M, Bullrich F, Croce CM: Frequent deletions and down-regulation of micro-RNA genes miR15 and miR16 at 13q14 in chronic lymphocytic leukemia. Proc Natl Acad Sci U S A 2002, 99:15524-15529.

14. Garzon R, Volinia S, Liu CG, Fernandez-Cymering C, Palumbo T, Pichiorri F, Fabbri M, Coombes K, Alder H, Nakamura T, Flomenberg N, Marcucci G, Calin GA, Kornblau SM, Kantarjian H, Bloomfield CD, Andreeff M, Croce CM: MicroRNA signatures associated with cytogenetics and prognosis in acute myeloid leukemia. Blood 2008, 111:3183-3189.

15. de Oliveira JC, Scrideli CA, Brassesco MS, Morales AG, Pezuk JA, Queiroz RP Yunes JA, Brandalise SR, Tone LG: Differential miRNA expression in childhood acute lymphoblastic leukemia and association with clinical and biological features. Leuk Res 2012, 36:293-298.

16. Rehmsmeier M, Steffen $P$, Hochsmann M, Giegerich R: Fast and effective prediction of microRNA/target duplexes. RNA 2004, 10:1507-1517.

17. The UniProt Consortium: The Universal Protein Resource (UniProt). Nucleic Acids Res 2009, 37:D169-D174.

18. Shields JM, Yang W: Identification of the DNA sequence that interacts with the gut-enriched Krüppel-like factor. Nucleic Acids Res 1998 26:796-802.

19. Zhang W, Geiman DE, Shields JM, Dang DT, Mahatan CS, Kaestner KH, Biggs JR, Kraft AS, Yang WW: The gut-enriched Krüppel-like factor (Krüppel-like factor 4) mediates the transactivating effect of p53 on the p21 WAF1/Cip1 promoter. J Biol Chem 2000, 275:18391-18398.

20. Rowland BD, Bernards R, Peeper DS: The KLF4 tumour suppressor is a transcriptional repressor of $\mathrm{p} 53$ that acts as a context-dependent oncogene. Nat Cell Biol 2005, 7:1074-1082.

21. Sherr CJ, Roberts JM: CDK inhibitors: positive and negative regulators of G1 phase progression. Genes Dev 1999, 13:1501-1512.

22. Kanai M, Wei D, Li Q, Jia Z, Ajani J, Le X, Yao J, Xie K: Loss of Kruppel-like factor expression contributes to SP1 over expression and human gastric cancer development and progression. Clin Cancer Res 2006, 12:6395-6402.

23. Kaul D, Mehrotra A: Functional characterization of AATF transcriptome in human leukemic cells. Mol Cell Biochem 2007, 297:215-220.

24. Yasunaga J, Taniguchi Y, Nosaka K, Yoshida M, Satou Y, Sakai T, Mitsuya H, Matsuoka M: Identification of aberrantly methylated genes in association with adult T-cell leukemia. Cancer Res 2004, 64:6002-6009.

25. Kluiver J, Poppema S, de Jong D, Blokzijl T, Harms G, Jacobs S, Kroesen BJ, van den Berg A: BIC and miR-155 are highly expressed in Hodgkin, primary mediastinal and diffuse large B cell lymphomas. J Pathol 2005, 207:243-249.

26. Eis PS, Tam W, Sun L, Chadburn A, Li Z, Gomez MF, Lund E, Dahlberg JE: Accumulation of miR-155 and BIC RNA in human B cell lymphomas. Proc Natl Acad Sci U S A 2005, 102:3627-3632.

27. Mattiske S, Suetani RJ, Neilsen PM, Callen DF: The oncogenic role of miR-155 in breast cancer. Cancer Epidemiol Biomarkers Prev 2012, 21:1236-1243.

28. Xu N, Papagiannakopoulos T, Pan G, Thomson JA, Kosik KS: MicroRNA-145 regulates OCT4, SOX2 and KLF4 and represses pluripotencyin human embryonic stem cells. Cell 2009, 137:647-658. 
29. Tian Y, Luo A, Cai Y, Su Q, Ding F, Chen H, Liu Z: MicroRNA-10b promotes migration and invasion through KLF4 in human esophageal cancer cell lines. J Biol Chem 2010, 285:7986-7994.

30. Schuetz A, Nana D, Rose C, Zocher G, Milanovic M, Koeniqsmann J, Blasig R, Heinemann U, Carstanjen D: The structure of the KLF4 DNA-binding domain links to self-renewal and macrophage differentiation. Cell Mol Life Sci 2011, 68:3121-3131.

31. Zhao W, Hisamuddin IM, Nandan MO, Babbin BA, Lamb NE, Yang WW: Identification of Krüppel-like factor 4 as a potential tumor suppressor gene in colorectal cancer. Oncogene 2004, 15:395-402.

32. Kharas MG, Yusuf I, Scarfone VM, Yang WW, Segre JA, Huettner CS, Fruman DA: KLF4 suppresses transformation of pre-B cells by $A B L$ oncogenes. Blood 2007, 109:747-755.

33. Yang WT, Zheng PS: Krüppel-like factor 4 functions as a tumor suppressor in cervical carcinoma. Cancer 2012, 118:3691-3702.

34. Boyum A: Isolation of mononuclear cells and granulocytes from human blood. Scand J Clin Lab Invest Suppl 1968, 21:77-89.

35. Laemmli UK: Cleavage of structural proteins during the assembly of the head of bacteriophage T4. Nature 1970, 227:680-685.

36. Raina A, Kaul D: LXR-a genomics programmes neuronal death observed in Alzheimer's disease. Apoptosis 2010, 15:1461-1469.

37. Larkin MA, Blackshields G, Brown NP, Chenna R, McGettigan PA, McWilliam H, Valentin F, Wallace IM, Wilm A, Lopez R, Thompson JD, Gibson TJ, Higgins DG: Clustal W and Clustal X version 2.0. Bioinformatics 2007, 23:2947-2948

38. Sali A, Blundell TL: Comparative protein modelling by satisfaction of spatial restraints. J Mol Biol 1993, 234:779-815.

39. Bowie JU, Lüthy $R$, Eisenberg D: A method to identify protein sequences that fold into a known three-dimensional structure. Science 1991, 253:164-170.

40. Laskowski RA, Moss DS, Thornton JM: Main-chain bond lengths and bond angles in protein structures. J Mol Biol 1993, 231:1049-1067.

41. Pettersen EF, Goddard TD, Huang CC, Couch GS, Greenblatt DM, Meng EC, Ferrin TE: UCSF Chimera-a visualization system for exploratory research and analysis. J Comput Chem 2004, 25:1605-1612.

42. DeLano WL: The PyMOL molecular graphics system. San Carlos, CA, USA: DeLano Scientific; 2010.

43. Vlahovicek K, Kajan L, Pongor S: DNA analysis servers: plot.it, bend.it, model.it and IS. Nucleic Acids Res 2003, 31:3686-3687.

44. De Vries SJ, van Dijk M, Bonvin AM: The HADDOCK web server for data-driven biomolecular docking. Nat Protoc 2010, 5:883-897.

45. De Vries SJ, Bonvin AMJJ: CPORT: a consensus interface predictor and its performance in prediction-driven docking with HADDOCK. PLOS One 2011, 6:e17695.

46. Gallivan JP, Dougherty DA: Cation-pi Interactions in Structural Biology. Proc Natl Acad Sci U S A 1999, 96:9459-9464.

\section{Submit your next manuscript to BioMed Central and take full advantage of:}

- Convenient online submission

- Thorough peer review

- No space constraints or color figure charges

- Immediate publication on acceptance

- Inclusion in PubMed, CAS, Scopus and Google Scholar

- Research which is freely available for redistribution

Submit your manuscript at www.biomedcentral.com/submit
Ciomed Central 